Abnehmer aus dem Unternehmensbereich müssen unter Nutzung von Netzwerkvorteile in der Lage sein, weitere vermittelbare Informationen aus der EMAS-Teilnahme zu erhalten.

- Die Öffentliche Hand als Nachfrager könnte ggf. die Kombination von EMAS und Produktlabel zum Standard für ihr Einkaufsverhalten machen und damit einen ,,pull“-Effekt auslösen.

- Die Aufsichtsbehörden können im Gegenzug zum Aufbau eines leistungsfähigen überprüfbaren Öko-Audit-Systems Deregulierungspotentiale nutzen.

- Von Seiten der EU müssen die entsprechenden Anreize gesetzt werden, die diesen Standard im Wettbewerb mit z.B. der ISO Norm stützen. EMAS als „Premium-ISO“ müßte zugleich derart transparent sein, daß auch die Unterschiede vermittelbar sind und sich somit Vorreiterunternehmen durch die Ausgestaltung von EMAS absetzen können. Dies könnte durch entsprechende Benchmarking-Aktivitäten wesentlich unterstïtzt werden.

\section{Anmerkungen}

(1) Kassebohm, K. et al.: Auditierung und Zertifizierung im Brennpunkt wirtschaftlicher und rechtlicher Interessen. In: ZfB (1994), S. $692-715$.

(2) Eger, T.: Wieviel Normung braucht der Markt? Ökonomische Aspekte der Vertragsfreiheit. In: Jahrbuch Ökonomie und Gesellschaft Nr. 11 (1995): Markt, Norm und Moral.

(3) Leveque, F.: Standards and standard-setting processes in the field of environment. In: R. Hawkins et al. (1995): Standards, Innovation and Competitiveness. The Politics and Economics of Standards in Natural and Technical Environments.

(4) Vgl. z.B. Sachverstündigenrat für Umwelffragen: Umweltgutachten 1998, Bundestags-Drucksache 13/10195, S.151ff.

(5) Clousen, J., U. Petschow, J. Behnsen: Umwelterklärungen als Innovationsbarometer. IÖW Schriftenreihe Nr. 114/97.

(6) Helbig, J. et al.: Wirtschaftspolitische Aspekte einer wachsenden Bedeutung von Normen im (betrieblichen) Umweltschutz. Tübingen 1997

\section{Der Autor}

Ulrich Peischow ist wissenschaftlicher Mitarbeiter am IÖW im Forschungsfeld Unweltökonomie und Umweltpolitik.

Kontakt: IÖW, Giesebrechistr. 13, 10629 Berlin. Tel. 030/88 45 94-0, Fax 030/882 54 39, E-mail: mailbox@ioew.b.eunet.de

Die Entwicklung und Implementation von EMAS auf EU-Ebene

\title{
Politikinnovation mit Hindernissen
}

\section{Mit der EMAS-Verordnung wurde die europäische Umweltpolitik um ein Kon- zept bereichert, das einige grundlegende Innovationen beinhaltet. Wie konnte sich EMAS im Prozeß der europäischen Politikformulierung durchsetzen? Wie effektiv ist die Implementation der Verordnung auf nationaler Ebene bisher?}

$D_{\text {sim }}$ Von Christoph Knill e Entwicklung von EMAS erfolgte im Kontext einer generellen strategischen Umorientierung in der europäischen Umweltpolitik, die sich seit Ende der achtziger Jahre beobachten läßt und speziell im fünften Umweltaktionsprogramm von 1993 nochmals bekräftigt wird. Im Kern dieser Trendwende steht die zunehmende Ersetzung und Ergänzung traditioneller hierarchischer Steuerungsinstrumente durch neue Regulierungsansätze, welche auf prozedurale Regulierung, Selbststeuerung, freiwillige Vereinbarungen und die Beteiligung der Öffentlichkeit abzielen (1). Wichtige Maßnahmen, die im Rahmen dieser neuen ,bottom-up“ Steuerung verabschiedet wurden, sind neben der EMAS-Verordnung die Richtlinien über den freien Zugang zu Umweltinformationen, die EcoLabel-Verordnung und die Rahmenrichtlinie zur Luftqualität.

Die Gründe für diese strategische Trendwende liegen hauptsächlich in den Implementationsdefiziten, welche sich im Rahmen der hierarchischen ,top-down“ Steuerung in verstärkter und andauernder Form gezeigt hatten. Diesem Problem soll mit der Verwendung von ,bottom-up“ Konzepten in zweifacher Hinsicht abgeholfen werden. Einerseits sind die neuen Instrumente von ihrem Design her institutionell weniger ,einschneidend", d.h. ihre Vorgaben sind "offener" und lassen sich somit leichter in bestehende regulative Arrangements auf nationaler Ebene integrieren. Administrative Widerstände bei der Implementation europäischer Vorgaben sind somit weniger wahrscheinlich. Zum zweiten sind die „bottom-up“ Ansätze speziell darauf ausgerichtet, die breite Unterstuitzung nationaler Akteure zu mobilisieren, d.h. es wird stärker auf die Interessenlage der Politikadressaten Bezug genommen. Je höher die Unterstiutzung ,von unten", so das Kalkuil, desto stärker ist wiederum der Druck auf nationale Implementeure, europäische Vorgaben effektiv umzusetzen. Die- se Annahmen werden im wesentlichen durch frühere Ergebnisse der Implementationsforschung bestätigt (2).

Allerdings sind auch ,bottom-up“ Instrumente trotz der institutionell weniger weitreichenden Vorgaben nicht frei von administrativen Implikationen auf nationaler Ebene und somit von Widerständen im Prozeß der Politikformulierung und Implementation.

\section{Umstriftene Einführung}

Die Entwicklung eines europäischen EMAS-Konzeptes basiert auf einem Vorschlag der Kommission, welcher wiederum von einer entsprechenden Initiative der Internationalen Handelskammer inspiriert war. Bereits während erster Diskussionen auf Kommissionsebene hatte sich gezeigt, daß die Mitgliedstaaten nicht bereit waren, die Einfuihrung von EMAS als rechtlich verbindliches Politikinstrument zu akzeptieren. Aufgrund dieser einhelligen Kritik seitens der Mitgliedstaaten entschloß sich die Kommission, EMAS als freiwilliges Instrument zu konzipieren. Als Folge dieses bereits im Vorfeld stark veränderten und nationalen Interessen entgegenkommenden Konzeptes fand der Vorschlag der Kommission breite Zustimmung im Ministerrat. Lediglich die Bundesrepublik sperrte sich zunächst vehement gegen die Maßnahme, während sich Großbritannien sehr schnell als stärkster Verfechter der Verordnung entpuppte. Wie bei vielen anderen Entscheidungen zur europäischen Umweltpolitik war damit auch die Entwicklung von EMAS durch das Aufeinanderprallen zweier gegensätzlicher nationaler Regulierungsvorstellungen geprägt.

Die wesentlichen Elemente der Verordnung entsprachen weitgehend dem flexiblen, auf prozeduralen Regeln und industrieller Selbstregulierung basierenden britischen Ansatz. Daher waren die mit der Maßnahme verbundenen institutionellen Anpassungskosten im Falle Großbritanniens sehr gering. Überdies hatten die Briten kurz zuvor einen eigenen nationalen Standard für Umwelt- 
managementsysteme entwickelt (British Standard 7750), der in vielen Bereichen als Grundlage des Kommissionsvorschlages gedient hatte.

Hingegen stand das europäische EMAS-Konzept in fundamentalem Widerspruch zur deutschen Regulierungsphilosophie. Insbesondere die deutsche Betonung von Stand der Technik und hierarchischer Intervention schienen nicht mit den prozeduralen, selbst-regulativen Elementen der Verordnung vereinbar. Vor dem Hintergrund dieser institutionellen Anpassungszwänge stieß der Vorschlag in Deutschland auf breite Ablehnung.

\section{Deutsche Bedenken}

Während das Umweltministerium das Konzept als $\mathrm{zu}$ wenig weitgehend und daher als regulativen Rückschritt charakterisierte, befürchtete die Industrie zusätzliche regulative Belastungen, welche durch die Einführung eines neuen und zusätzlichen Politikinstruments entstehen könnten. Auch der Einwand, daß EMAS lediglich als freiwilliges Instrument konzipiert war, vermochte deutsche Gemüter wenig $\mathrm{zu}$ beruhigen. So wurde erwartet, daß sich die ursprüngliche Freiwilligkeit sehr schnell zu ,freiwilligem Zwang“ entwickeln würde, wenn Versicherungen und Banken die Unternehmen dazu drängen, Umwelt-Audits durchzufiihren, um eventuellen Schäden und damit verbundenen finanziellen Verantwortlichkeiten vorzubeugen.

Trotz ihrer starken Bedenken gaben die Deutschen nach vergleichsweise kurzer Verhandlungsdauer ihren ursprünglichen Widerstand auf und stimmten der Verordnung zu. Diese überraschende Wendung läßt sich vor allem auf zwei Faktoren zurückführen:

Einerseits antizipierte die Bundesrepublik bevorstehende Änderungen des Abstimmungsverfahrens im Ministerrat durch den Vertrag von Maastricht. Wenn die Bundesrepublik bei der sich abzeichnenden qualifizierten Mehrheitsregel noch in irgendeiner Weise Einfluß auf die Gestaltung der Verordnung nehmen wollte, war sie zur Kooperation mit den anderen Mitgliedstaaten gezwungen.

Ein zweiter Aspekt, der den Deutschen ihre Zustimmung erleichterte, waren gewisse Zugeständnisse im Hinblick auf eine stärkere Technologieorientierung der Verordnung. Eine erfolgreiche Teilnahme an EMAS setzt somit nicht nur voraus, daß ein Betrieb umweltorientierte Management-Praktiken anwendet, sondern auch, das er durch die Anwendung bestverfügbarer und ökonomisch vertretbarer Technologien an einer kontinuierlichen Verbesserung des betrieblichen Umweltschutzes arbeitet.

\section{- Effektive Implementation?}

Betrachtet man die Umsetzung der EMAS-Verordnung auf nationaler Ebene, so zeigt sich - oberflächlich betrachtet - das Bild einer relativ effektiven Implementation. Die formale Umsetzung der Verordnung auf nationaler Ebene ist generell in Einklang mit den europäischen Vorgaben erfolgt. Auch im Hinblick auf die praktische Umsetzung zeigt sich ein relativ positives Bild. In den meisten Mitgliedsländern sind inzwischen die administrativen und institutionellen Voraussetzungen für ein nationales EMAS-System geschaffen worden. Ein wichtiger Grund für dieses, verglichen mit ,top-down“ Konzepten, relativ effektive Bild formaler und praktischer Umsetzung liegt darin, daß die Verordnung nicht das Ersetzen bestehender nationaler Regulierungskonzepte verlangt, sondern letztere um ein weiteres Instrument ergänzt. Der institutionelle Anpassungsdruck der Maßnahme auf nationale Strukturen ist damit wesentlich geringer.

Das bisher präsentierte Muster einer durchweg effektiven Implementation relativiert sich jedoch, wenn man die auf nationaler Ebene erfolgten institutionellen und administrativen Anpassungen genauer hinterfragt. Hierbei lassen sich drei Problembereiche unterscheiden.

Erstens zeigt insbesondere der Fall der deutschen Umsetzung, daß die wenig spezifizierten prozeduralen Vorgaben der Verordnung und der damit verbundene nationale Handlungsspielraum Probleme nach sich ziehen können. Wenngleich die Bundesrepublik, im europäischen Entscheidungsprozeß noch als Bremser in Erscheinung getreten, derzeit einen absoluten Spitzenplatz einnimmt, wenn man die Anzahl der zertifizierten Betriebe betrachtet, verweisen Kritiker auf die relativ industriefreundlich gestalteten Verfahrensregeln der deutschen EMAS-Validierung. So wird ein wichtiger Grund für die hohe Zahl teilnehmender Unternehmen im System der Umweltgutachter gesehen. Im Gegensatz zu Großbritannien basiert das deutsche Validierungssystem auf der Begutachtung durch individuelle Umweltgutachter und nicht etwa durch Prüfungsorganisationen oder -kommissionen. Dieses System wurde mehrfach als unzureichend kritisiert, da kaum vorausgesetzt werden kann, daß ein individueller Um- weltgutachter alle notwendigen Kompetenzen für diese komplexe Aufgabe besitzt. Ein weiterer Umstand, der den deutschen ,Erfolg“" erklärt, liegt in dem starken Einfluß der Industrie innerhalb des Umweltgutachterausschusses, der wichtige regulative Funktionen im Rahmen des deutschen EMAS-Systems wahrnimmt (3).

\section{- Fehlende umweltrechtlich Integration}

Ein zweites Problem der Implementation von EMAS besteht in der bisher nur unzureichend oder überhaupt nicht erfolgten Integration in umweltrechtliche Genehmigungsverfahren. So ist bisher nicht geklärt, inwieweit registrierten Unternehmensstandorten entsprechende Erleichterungen bei der Genehmigung und Überwachung industrieller Anlagen gewährt werden sollen. Zwar werden in vielen Staaten entsprechende Erleichterungen diskutiert, etwa im Hinblick auf eine Beschleunigung des Verfahrens oder der Selbstkontrolle bei der Emissionsuiberwachung, konkrete Schritte sind bisher jedoch nicht erfolgt. Aufgrund dieser Unsicherheit fehlt vielen Unternehmen der Anreiz, sich an EMAS zu beteiligen. Besonders gravierend wirkt sich diese fehlende Abstimmung mit anderen Verfahren bisher im Falle Frankreichs aus. Aufgrund fehlender institutioneller und prozeduraler Arrangements zur Klärung des Zusammenhangs von Genehmigungsverfahren und EMAS konzentriert sich der Großteil der an einer Validierung interessierten Unternehmen auf den ISO-Standard 14001 (4). Aber auch in der Bundesrepublik und Großbritannien ist - trotz entsprechender Ankündigungen - eine umweltrechtliche Integration von EMAS bisher ausgeblieben. In Großbritannien ergaben sich diesbezügliche Verzögerungen aufgrund nationaler Verwaltungsreformen (5).

Drittens wird der Erfolg von EMAS durch eine unzureichende Abgrenzung zwischen EMAS und anderen Umweltmanagementsystemen beeinträchtigt. Die fehlende Abgrenzung und Klarstellung der Reichweite, Voraussetzungen und Bedingungen unterschiedlicher Zertifizierungssysteme hat in vielen Ländern eine eher abschreckende Wirkung auf die Industrie ausgeübt. Angesichts dieser Unsicherheit haben sich viele Unternehmen für das weltweit anerkannte ISO-System entschieden. Vor allem dieser Aspekt erklärt das verglichen zu Deutschland relativ geringe Interesse an EMAS seitens der britischen Industrie, was vor dem Hintergrund des weiter oben beschriebenen 
europäischen Entscheidungsprozesses überraschend erscheint. Auch die französische Industrie bevorzugt den ISO Standard.

Insgesamt zeigt der Fall von EMAS, daß auch die sog. neuen Instrumente der europäischen Umweltpolitik durchaus nicht frei von Implementationsproblemen sind. Wie die obigen Ausführungen verdeutlichen, können $\mathrm{zu}$,offene“ europäische Vorgaben dazu führen, daß auf nationaler Ebene nicht genügend getan wird, um den Zielen europäischer Politik gerecht zu werden. Umgekehrt bewirken diese Defizite gleichzeitig, daß die Politikadressaten, deren Unterstützung eine effektive Implementation voraussetzt, ihr Interesse verlieren bzw. die Maßnahme nicht in ausreichender Weise unterstiitzen. Neue Instrumente wie EMAS sind somit nicht nur aus der regulativen, sondern auch aus der Implementationsperspektive lediglich als Ergänzung und nicht als Alternative zu traditionellen Instrumenten zu sehen.

\section{Anmerkungen}

(1) Héritier, Adrienne, Christoph Knill, Susanne Mingers: Ringing the Changes in Europe. Regulatory Competition and the Transformation of the State. Berlin 1996.

(2) Mayntz, Renate: Implementation von regulativer Politik. In: Renate Mayntz (Hrsg.), Implementation politischer Programme II, Opladen 1983, S.50-74.

(3) Lenschow, Andrea: The Implementation of EU Environmental Policy in Germany. In: Christoph Knill (Hrsg.): The Impact of National Administrative Traditions on the Implementation of EU Environmental Policy. Research Report. Florence: European University Institute.

(4) Knill, Christoph: Appropriate Implementation. The Impact of National Administrative Traditions on European Policy-Making. In: Journal of Public Policy, Vol. 18 (1998), №.1.

(5) Knill, Christoph, Andrea Lenschow: Compliance with Europe: The Implementation of EU Environmental Policy and Administrative Traditions in Britain and Germany. Erscheint in: Journal of European Public Policy, Vol. 5 (1998), №. 4.

\section{Der Autor}

Dr. Christoph Knill ist wissenschufflicher Milarbeiter an der Max-Planck-Arbeilsgruppe Rechi der Gemeinschafisgüter.

Kontakt: Poppelsdorferallee 45, 53115 Bonn, Tel. $0228 / 91416-0$

Die Position der EU-Kommission zur Revision der EMAS-Verordnung

\title{
Aus den Erfahrungen lernen
}

\author{
Aus der bisherigen Praxis des EG-Öko-Audit-Systems hat sich eine Fïlle von \\ Veränderungsbedarf ergeben. Basierend auf der Konsultation aller Beteiligten \\ wird die DG XI der EU-Kommission einen Revisionsvorschlag vorlegen, der dem \\ Rechnung trägt.
}

$\mathrm{D}$ Von Klaus Krisor ie Europäische Gemeinschaft betreibt seit etwa 25 Jahren Umweltpolitik. Die bisherigen Aktionen der Gemeinschaft und der Mitgliedstaaten auf dem Gebiet des Umweltschutzes basierten fast ausschließlich auf dem Erlaß von Rechtsvorschriften (Command and Control Approach). Wir brauchen in Zukunft eine breitere Palette von Instrumenten. Dazu gehören marktorientierte Instrumente, die auf dem Prinzip der Anwendung ökonomischer und steuerlicher Anreize beruhen. Das erfolgreichste Instrument in dieser Gruppe ist das Öko-Management/ Öko-Audit-System (EMAS), das sowohl die Unterstiitzung der Unternehmen als auch die der Umweltschützer hat. EMAS ist seit 1993 in Kraft. Artikel 20 der EMAS-Verordnung sieht vor, daß die Kommission die jetzige Verordnung anhand der bei ihrer Durchfiihrung gemachten Erfahrungen überprüft und dem Rat gegebenenfalls geeignete Änderungen vorschlägt. Dies gilt insbesondere im Hinblick auf eine Ausdehnung des Systems auf andere Branchen. Dieser Prozeß läuft gerade.

\section{Grundprinzipien der Revision}

Es ist noch nicht sicher, wie der neue Vorschlag aussehen wird, einige Grundprinzipien sind jedoch bereits heute deutlich:

1. Die neue EMAS-Verordnung wird auch weiterhin ein freiwilliges Instrument sein.

2. Es wird keine Änderung der grundlegenden Bestimmungen der Verordnung geben, d.h. die teilnehmenden Unternehmen brauchen keine neuen oder andere Bestimmungen zu erfiillen, um auch in Zukunft teilnehmen zu können (Kontinuität fïr EMAS-registrierte Betriebe).

3. Wir werden uns auf denjenigen Gebieten enger an die ISO Normen der Reihe 14000 anlehnen, auf denen diese Normen gleich hohe Anforderungen an den Umweltschutz stellen wie EMAS. Die logischere Struktur der ISO Normen wird für gewisse Teile (Anhänge) der Verordnung übernommen werden, um eine größere Kompatibilität mit ISO zu erreichen. Dies wird zu einer Vereinfachung der Struktur des Systems führen. Ziel ist es, daß ein EMAS-registriertes Unternehmen automatisch ein ISO Zertifikat bekommt, da es alle Bedingungen für dieses Zertifikat erfïllt.

4. Die Verordnung wird auf neue Sektoren erweitert werden. Bisher können am EMASSystem alle gewerblichen Branchen teilnehmen, die in der Verordnung genannt sind. Es handelt sich im wesentlichen um das produzierende Gewerbe einschließlich Energieerzeugung und Recyclinggewerbe. In Zukunft sollen an EMAS alle Branchen teilnehmen, insbesondere auch der Dienstleistungssektor.

Neue Sektoren sind: der Handel, das Transportgewerbe, das Baugewerbe, Verteilernetze für Wasser, Gas und Strom, Land- und Forstwirtschaft, das Dienstleistungsgewerbe, Banken, Versicherungen, Krankenhäuser, Tourismus und Freizeitgewerbe, Hotels und Golfklubs und, nicht zu vergessen, die öffentliche Verwaltung.

Wahrscheinlich wird diese Ausdehnung großzügig sein und alle Branchen umfassen. Es geht dabei um die Frage: Wie muß die Öko-Audit Verordnung verändert werden, damit sie auf andere Branchen anwendbar ist? Die Definition des Standorts spielt hierbei eine besondere Rolle: wie kann man z.B. den Standort beim Transportgewerbe definieren?

\section{Vorteile für die Teilnehmer}

5. Das neue System soll einen deutlichen Mehrwert für teilnehmende Unternehmen bringen. Damit sich das EMAS-System besser am Markt durchsetzen kann, muß es attraktiv sein, insbesondere auch für kleine und mittelständische Unternehmen. Obwohl EMAS ein freiwilliges System ist, wird die Teilnahme in Zukunft für fortschrittliche Unternehmen eine Notwendigkeit sein. Experten sind überzeugt, daß EMAS eine strategische Notwendigkeit für den Unternehmenserfolg sein wird. Schon kurzfristig macht sich ein Umweltmanagementsystem bezahlt, $d a$ folgende Vorteile zu erwarten sind: 
(c) 20I0 Authors; licensee IÖW and oekom verlag. This is an article distributed under the terms of the Creative Commons Attribution Non-Commercial No Derivates License (http://creativecommons.org/licenses/by-nc-nd/3.o/), which permits unrestricted use, distribution, and reproduction in any medium, provided the original work is properly cited. 